Proceedings of the ASME 2020 Conference on Smart Materials, Adaptive Structures and Intelligent Systems

SMASIS2020

September 14-16, 2020, Irvine, CA, USA

SMASIS2020-17491

\title{
EXPERIMENTAL STUDY OF FLEXIBLE SKIN DESIGNS BETWEEN A MOVING WING SEGMENT AND A FIXED WING PART ON A FULL SCALE DEMONSTRATOR
}

\author{
Martin Radestock ${ }^{1}$, Johannes \\ Riemenschneider \\ German Aerospace Center (DLR) \\ Institute of Composite Structures \\ and Adaptive Systems \\ Braunschweig, Germany
}

\author{
Alexander Falken \\ INVENT GmbH \\ Braunschweig, Germany
}

\author{
Johannes Achleitner \\ Technical University Munich \\ Institute of Aircraft Design \\ Munich, Germany
}

\begin{abstract}
Commercial aircraft today require efficient high-lift and control systems on the wings to reduce the drag in flight or decrease the take-off and landing speeds. Morphing mechanisms are one approach for improved high-lift systems. In most cases the objective function is an increased lift to drag ratio or the noise reduction. On closer examination control systems as well as morphing mechanisms are located in a certain wing segment. The transition between a moving wing part and the fixed wing is a step, which creates additional vortices. This segments the wing in span-wise direction and reduces the efficiency. A flexible skin between a moving and a fixed wing parts smooths the contour and minimize the efficiency reduction of the wing. A full scale demonstrator of a wing segment was manufactured with two flexible skin designs.

The first subcomponent connects a morphing leading edge with a rib of the wing over a span of one meter. The skin is a material mix of ethylene-propylene-diene monomer (EPDM) rubber and fiberglass-reinforced plastic. The rubber is the basis of the skin and the glass-fiber is added as local skin stiffeners in the form of strips in chord-wise direction. The second subcomponent blends the aileron with a rib of the wing in a triangular design. The connection of three different hinges realizes a morphing triangle, which is loaded in an in-plane shear only state of stress in each aileron position. The core of the triangle is a $3 D$ printed structure, which is free in shear. The covering skin is a combination of EPDM with carbon fibers oriented in $+/-30^{\circ}$ direction to obtain shear compliance and to resist the loads on the triangle.

The deformation of each concept is identified at the demonstrator. Therefore, an optical measurement system scans the surface in the initial and deflected state. The required deformation precision of the concepts differs due to their
\end{abstract}

\footnotetext{
${ }^{1}$ Contact author: Martin.Radestock@dlr.de
}

design. The contour at the leading edge requires a certain shape over the span. The analysis of the skin buckling is one requirement at the transition triangle during the aileron motion.

The experimental results show a smooth transition contour at the leading edge and no buckling effects at the triangle. The results can be used for the validation of simulation models. Furthermore, both skin concepts cover the gap between a moving wing segment and a fixed wing part. The elimination of steps in span-wise direction can improve the aero-acoustic behavior along the wing for future aircraft.

Keywords: gapless wing, Shape adaptive transition skin, GFRP elastomer composite 


\section{INTRODUCTION}

The wings of aircraft are complex structures with multifunctional components. These include airbrakes, spoiler, high lift systems such as flaps and slats or control surfaces such as ailerons. The position of all these components is on a specific wing segment in span-wise and chord direction. In general there is no transition between these moving parts and the fixed wing along the span. Especially these gaps contribute to the overall noise of an aircraft. The noise of flap edges was measured and predicted in the work of Brooks and Humphrey [1]. Therefore, a gap cover or a smooth transition skin can reduce the noise.

An example is the transition skin at a flap with the Flexsys morphing system [2]. This concept has already been tested in flight tests and the aim of the transition skin is to reduce the turbulent airflow and the resulting noise. Hutcheson et al. also investigate the reduction of noise by a transition skin between a flap and the fixed wing [3]. Depending on the Mach number and flap deflection, a noise reduction of 5 to $17 \mathrm{~dB}$ can be achieved with a gap covering. In the work of Woods et al. an aerodynamic simulation for an UAV wing shows the streamlines for a flap with and without transition [4]. The freestream velocity is $87 \mathrm{~m} / \mathrm{s}$. With a transition the lift coefficient increases by approximately $4 \%$ and the drag coefficient is reduced by $3 \%$.

Covering the gap between a moving and a fixed wing part is an essential step for quiet aircrafts. This is also a question for morphing wings. The number of concepts is enormous and also summarized in literature of Barbarino et al. or Vasista et al. $[5,6]$.

Especially camber morphing concepts have the same problems as conventional control surfaces or high lift systems. They are located at a certain wing segment and the transition to the fixed wing is not considered.

This study presents two covering concepts, which were realized at a full scale demonstrator. The first concept is a transition skin with a span of one meter between a fixed wing part and morphing leading edge. The morphing leading edge is similar to the work of Kintscher et al. [7]. The second covering concept closes the gap between an aileron and a fixed wing. The challenge is a high flexibility in different directions and a high stiffness in other direction.

The main objective is the deformation analysis of the two covering concepts on the demonstrator. Therefore, an optical measurement system scans the surface of the skin in different deflection positions. The morphing leading edge deflects only from an initial position to a droop configuration in order to replace the slat function. The aileron moves in upward and downward direction during the measurement. The results are the deflections only in the end positions, because the system cannot track the skin deformation during the motion.

The skin at the leading edge concept is comparable to the simulation model with a maximal deviation of 12 millimeters. The cover at the aileron cannot be compared to the simulation, because the out of plane deflection is approximately 0.05 millimeter in the simulation while the shapes deviate by eight millimeters due to orientation of the different measurements. The two gap covering concepts show the reliability at a demonstrator and can be the first step towards quite aircrafts.

\section{EXISTING CONCEPTS FOR TRANSITIONS SKINS}

Next to the mentioned concepts from Flexsys and Woods et al. several concepts exists to close the gap between a moving wing segment and a fixed rib. Different granted patents show ideas from industry and research groups [8-12]. In the concept of Kunz an additional flap is added, which is oriented perpendicular to the main flap in span wise direction [12]. This additional flap slides in the main flap during the motion. The implementation of such an additional flap leads to another complex kinematic in the wing. The patents of Diller et al. and Caton et al. have a similar basic concept as different versions $[8,9]$. The skin of the transition is a silicone elastomer skin with embedded rods. The number of the rods in the skin is high and they slide in holes of the skin. The sliding is necessary, because the silicone skin captures the strains during the deformation, but the rods cannot handle the strains. Due to the high expected number of sliding rods the friction adds additional required forces to this concept. In the concept of Etling rib segments are used in the transition region [10]. Two rods in span wise direction connect the ribs. The motion of the rods defines the shape of the transition skin for a flap [10]. The concept does not have a connection between the ribs and the skin. This raises the question how the skin can be flexible and carry the aerodynamic loads at the same time. Another skin approach suggests the work of Khorrami with an elastomer skin and different cores types in a transition rectangle [11]. The three core types are a solid elastomer rectangle, elastomer skins with a hollow core and elastomer skins with a foam core. A wind tunnel experiment was done for this concept and shows a reduction of approximately $3 \mathrm{~dB}$ over a wide frequency range for the deflected flap with gap cover compared to a flap without cover [13]. The work of Sreekantamurthy pick up the concept from Khorrami for simulation studies [14]. The elastomer skin with the hollow cores shows high skin strains of approximately $500 \%$. Also the high deformations lead to wrinkling of the transition surface. The work of Streett describes a Continuous mold-line link (CML) as a gap cover concept for a fowler flap [15]. This concept was tested in an wind tunnel experiment where about $30 \%$ of the flap span is replaced by the CML [16]. The gap cover reduces the noise by $6-8 \mathrm{~dB}$ at a free stream Mach number of 0.22 .

\section{WING BASE FOR GAP COVER CONCEPTS}

The gap covers are application-oriented for an aircraft wing. Figure 1 shows a DLR aircraft configuration similar to an A320 [17]. The zoom shows the morphing leading edge, the aileron and the two covering concepts in detail. The span of the transition skin is one meter and the chord length is approximately 0.4 meter. The span of the gap cover triangle is about 0.75 meter at its widest point and has a chord length of 0.45 meter. 


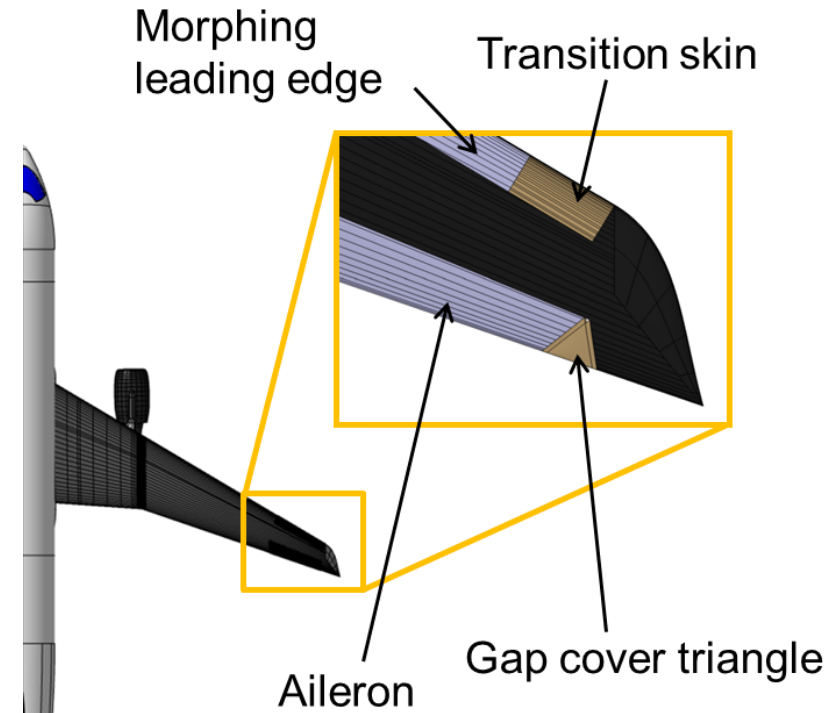

$\longrightarrow$

Figure 1: DEFINTION OF GAP COVER POSITIONS AT THE WING

\section{TRANSITION SKIN CONCEPT AT THE LEADING EDGE}

The morphing leading edge replaces the function of a slat. The calculated deflection is a result of an in-house framework, which is summarized in the work of Kintscher et al. [7]. The profile droops the nose down by $13^{\circ}$ when actuated. This angle is a result between two lines. Each line connects nose point and the spar point from the chord line. One line is from the initial position and the other line is from the morphed position.

Figure 2 shows a possible deformation of a transition skin with a span of one meter and the chord line angle change by $13^{\circ}$. The far right green section represents the deformed morphing leading edge, while the far left green section is the connection to a fixed rib that merges into the winglet. This surface is only a CAD model in and not the result of an aerodynamic optimization. A spline from the far left section to the far right section connects the nose points of each segment. As shown in Figure 2, the nose points of the outer sections differ from each other, because the deformation of the morphing leading edge is a rolling motion and not a pure bending of the nose. Otherwise, a slotted droop nose is required to perform the morphing. The rolling motion of the morphing leading edge reduces the maximum strain in skin and also reduces the required strains in the transition skin.

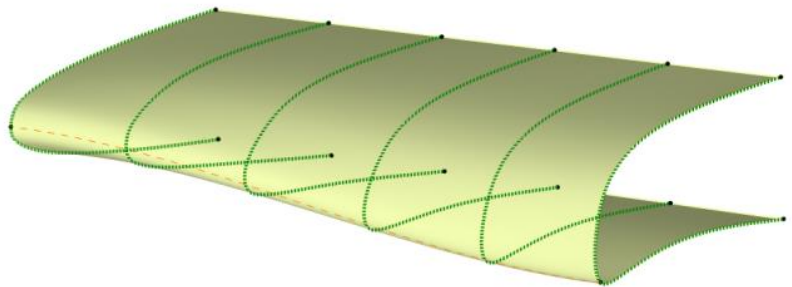

Figure 2: 3D CAD MODEL OF THE TRANSITION SKIN

Figure 3 shows the structural concept of the transition skin at the leading edge. The materials are the synthetic rubber ethylene propylene diene monomer (EPDM) and glass fiber reinforced plastic (GFRP). The base of the skin is the EPDM, which is visible in the section A-A. The GFRP is placed as stripes along the chord direction of the profile. These stripes are located on the outer and the inner transition skin surface.

The basic idea is a variation of the GFRP stripes in dimensions and position in order to achieve a variable stiffness along the span. In this case, only the position of the stripes varies and the thickness remains constant at one millimeter. In total, there are 24 GFRP stripes in the skin on inside and outside.

The first millimeter of the GFRP from the spar interface connects the GFRP stripes on the outer skin surface. The stripes can be manufactured simply by cutting out GFRP layers that covers the entire transition skin using a CNC prepreg cutter. So, the position of the GFRP stripes is exact. This is similar for the GFRP stripes at the inner skin contour. The structure is a shellonly structure, this means no additional components as stringers, frames or ribs are attached to the inner skin surface in the transition area.

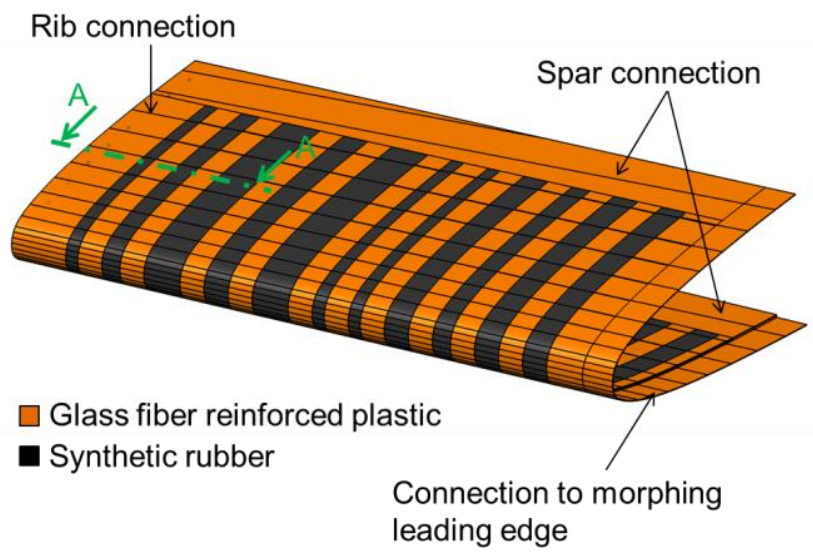

Section A-A

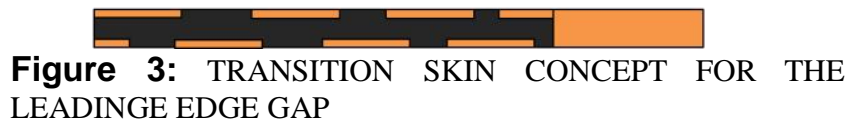

Figure 3 also shows the skin design for the connection to the fixed wing parts. The skin consists only out of GFRP layers 
at the interfaces to the rib, the spar and the morphing leading edge. Especially the rib and the spar connection require to be made solely from GFRP, because these parts are screwed to the fixed wing parts at the demonstrator. If there is EPDM in the connection area, the screws will squeeze the rubber material locally and failures may occur.

The skin concept has the potential for a full integration to a wing, as the outer GFRP layers can be part of adjacent components. The connection to the morphing leading edge already shows such integration at the demonstrator. The first millimeter of the transition skin is extruded in span wise direction by 10 millimeters towards the morphing leading edge. This is an approximate section of morphing leading edge for the demonstrator. The leading edge from the in-house framework has a specific GFRP layup. This layup can be applied directly to the extruded transition skin. The outer surface between transition skin and morphing leading edge is continuous.

\section{GAP COVER TRIANGLE CONCEPT AT THE AILERON}

The basic triangle as a gap cover derive from previous works $[18,19]$. The cover is intended for control surfaces on an unmanned aerial vehicle (UAV). This concept is consistently developed from the UAV control surfaces to an aileron for the full-scale demonstrator.

Figure 4 shows the basic concept and the parts of the gap cover triangle. The concept idea is that the three red dashed dotted hinge lines cross in a single point. The hinge axes are defined by the aileron axis and the axes of two flexible hinge parts. The result is that the purple triangle is loaded in a pure in-plane shear stress state.

The gap cover triangle consists of three parts. These parts are the hinges as H-beams, a 3D printed core and a covering skin of the core. A cover of the H-beams is not shown in Figure 4 , but it is required for the application. Such covers would be attached to the aileron and to the fixed wing. These covers slide on top of the covering skin of the core.

The core of this concept is 3D printed. One essential requirement is a free degree of freedom in shear. So, the core does not add any additional stiffness when the core is extended in span-wise direction due to the aileron motion. This is realized by the special design of the ribs and their crossing points. The ribs blend in two directions and are parallel to the H-beams. The ribs cross each other at several points, but they are not connected to each other in order to obtain a free inplane shear motion. At a crossing point, the ribs intersect each other without being connected. This is implemented for all crossing points and it is also done at different heights of the core. Due to the core design the ribs cannot be disassembled from each other. Therefore, the core manufacturing requires a $3 \mathrm{D}$ printing process. In the printing process the crossing ribs have a small support structure, which is designed as a weak link and to be cracked manually before the first application.

The covering skin of the core has similar requirements as the core. It is essential that the skin does not significantly increase the shear stiffness of the triangle. Otherwise the free in shear property of the core vanishes. On the other hand the aerodynamic loads act on top of the skin and therefore a minimum out of plane bending stiffness is required. Figure 4 shows the material combination of the skin cover in a small box. The skin is made of EPDM with carbon fiber epoxy filaments.

The carbon fibers are aligned at $+/-30^{\circ}$ angle, parallel to the H-beam hinge part sidewalls. The angle is a result of a simulation study for different fiber angles in the EPDM. The positive and the negative angle are always equal, so that the fibers in the skin are symmetrical. The material design is done with a two-scale FEM simulation approach consisting of a microscale repeated unit cell model and a macro scale global model, where the smeared material properties obtained from the micro scale model have been applied. Tensile tests and shear deformation tests of specimen validate the material properties obtained from the micro scale simulation. At the angle of $30^{\circ}$ the skin shows the best shear compliance.

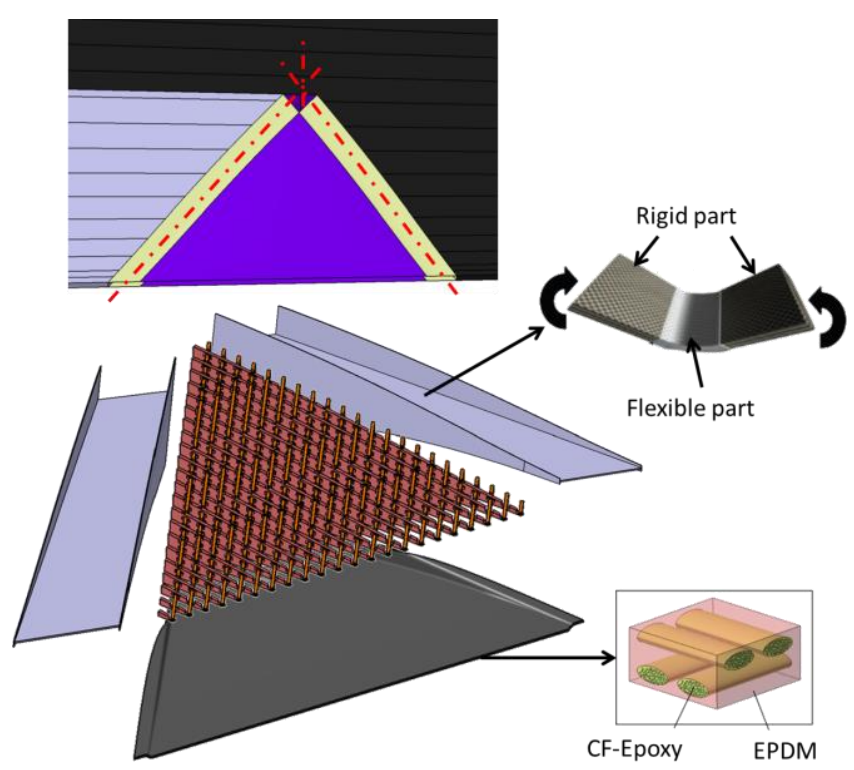

Figure 4: GAP COVER TRIANGLE CONCEPT FOR THE AILERON

The two hinges in Figure 4 are H-beams. One hinge is attached to the aileron and the other one to the fixed wing. The core is only placed in the triangle during the assembly process. The covering skins are attached to the hinges and force the motion of the core.

The beam materials are carbon fiber reinforced plastic (CFRP) and EPDM. The connection interfaces of the H-beams are a stiff CFRP-only structure while the middle part of the beams remains flexible. Therefore, the CFRP layers in the middle part are not continuously. The thickness of CFRP reduces from two millimeter down a single layer of CFRP. EPDM covers the discontinuous layers of CFRP shown in Figure 5. The design of CFRP layers was investigated for different bending probes in a test rig. The main objective of the 
experimental investigations is to maximize the allowable bending angle of the hinge without plastic deformation.

Further details about the hinges and the experimental investigations are specified in detail the $\mathrm{PhD}$ thesis of Krollmann and the conference article of Krollmann et al. [20,21].

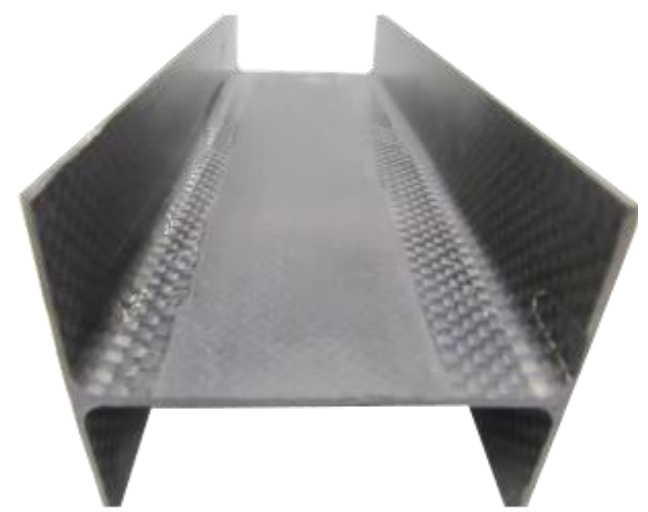

Figure 5: GENERAL DESIGN OF H-BEAM HINGES

\section{DEMONSTRATOR DESIGN}

The demonstrator design contains only a segment of the wing model. Figure 6 shows the outer part of the wing and the red dotted lines mark the realized wing segment. The winglet is cut off due to manufacturing simplifications. The cutoff at the leading edge towards wing root follows the rectangular form of the transition skin. This simplifies the manufacturing of the transition skin and an attached morphing leading edge. The angle is a result of the swept wing in the original model in Figure 1. The aileron cutoff has a similar angle so that the trailing edge and the cutoff line are perpendicular. Only the wing box cutoff is parallel to the winglet cutoff.

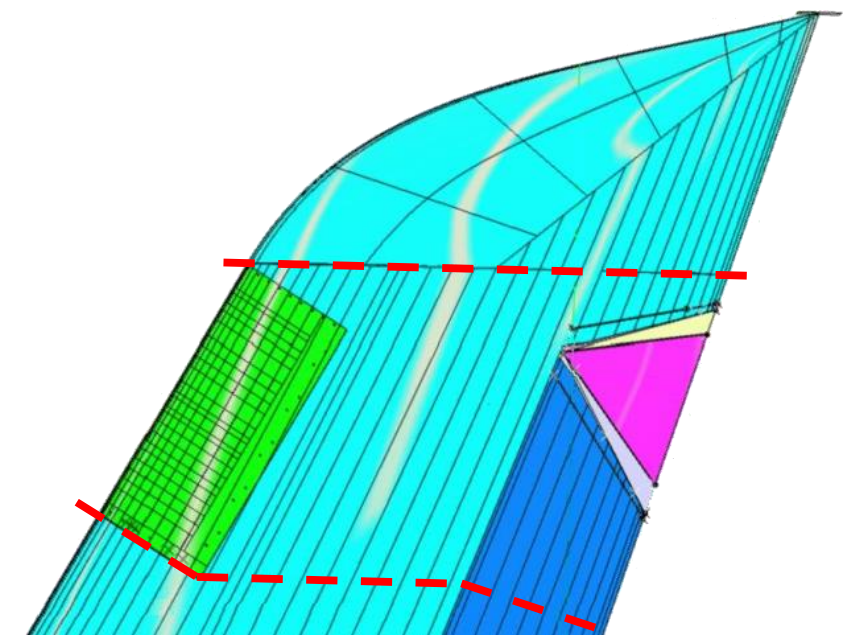

Figure 6: CAD MODEL FOR DEMONSTRATOR SEGMENT FROM SUCTION SIDE

Figure 7 shows the image of the complete demonstrator. The transition skin at the leading edge has a clear paint stripe, which goes from pressure side to suction side along the span as a curve. Therefore, only half of the GFRP stripes and the EPDM composition from Figure 3 are visible. The leading edge deformation is not completely achieved by the demonstrator due to the white paint at the leading edge. A simulation model shows for the $13^{\circ}$ chord line angle change result in approximately $5 \%$ strain in the skin. The paint does not tolerate such high strains and develop cracks. Therefore, the deformation is reduced to $80 \%$ of the required deformation at the demonstrator. Nevertheless, the EPDM in the skin can handle these strains.

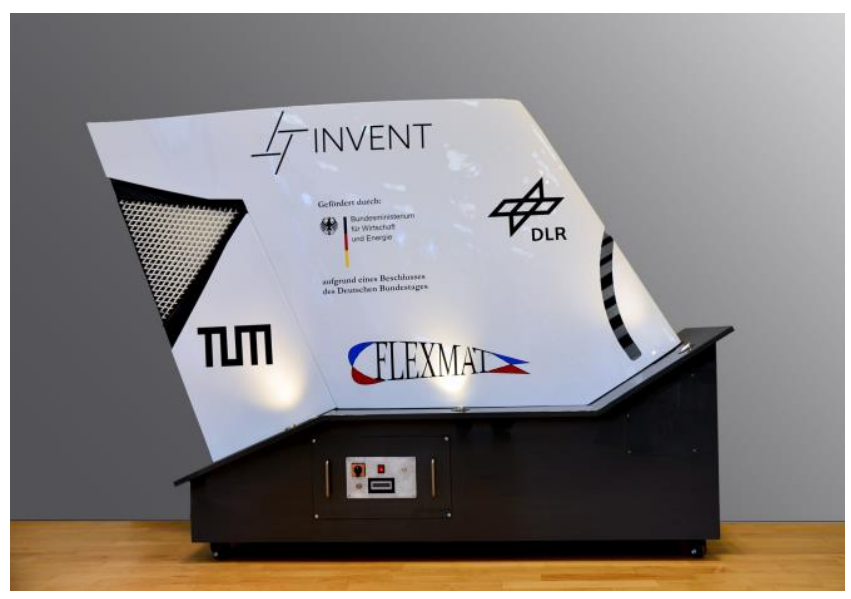

Figure 7: FLEXMAT DEMONSTRATOR WITH VIEW ON PRESSURE SIDE

The gap cover triangle in Figure 7 is not complete due to demonstration purposes. So, the 3D printed core is visible from the pressure side and one covering skin is attached on the suction side of the demonstrator. The core must be glued to the $\mathrm{H}$-beams due to the missing skin on the pressure side. That is different compared to the concept description. Otherwise the core is not forced to move due to the surrounding constraints.

Also, the H-beams need an additional covering, because the EPDM skin with CFRP fibers covers only the core. Such covers can be flexible GFRP stripes, which are attached to the fixed wing and the aileron. These covers slide over the skin. The demonstrator has no such covers, because they will disturb the measurement. The measurement determines the deformation of the core covering skin and the deformation of the H-beam hinges. In order to measure both at the same time, the measurement system scans the suction side of wing segment.

Each gap cover concept has a separate motor. The transition skin at the leading edge has a rotating stepping motor with approximately $5 \mathrm{Nm}$ and a spindle drive in order to transform the rotation to a linear actuation at the leading edge. A brushless servomotor with roller screw powers directly the aileron as a linear actuator. The actuator has a maximal linear force of approximately $2 \mathrm{kN}$ and is oversized for the required force at the aileron. 


\section{SIMULATION RESULTS FOR TRANSITION SKIN}

The simulation results of the transition skin are the comparison base for the experiment. Figure 8 shows the total deformation of the transition skin at the leading edge. The skin has a fixed bearing at the spar and the rib as boundary conditions. Also, the arrow indicates the load introduction point for the morphing leading edge. In this case only a single force represents the actuation of the morphing leading edge. This is a simplification compared to the literature of Kintscher et al. where 4 load introductions points are used [7]. A single force at the morphing leading edge does not produce a perfect shape of the deformed leading edge, but the angle change of the chord line with about $13^{\circ}$ can be achieved.

The simulation is a static-mechanic simulation in ANSYS Workbench. The total deformation of 55 millimeters represents the complete angle variation of the chord line. The nearly continuous deformation shows that the stiffness distribution is also continuous. There are no high stiffness gradients along the span, which would lead to high local deformation. Further information on the simulation is in literature, where a span wise segment of the wing is simulated and experimentally validated [22].
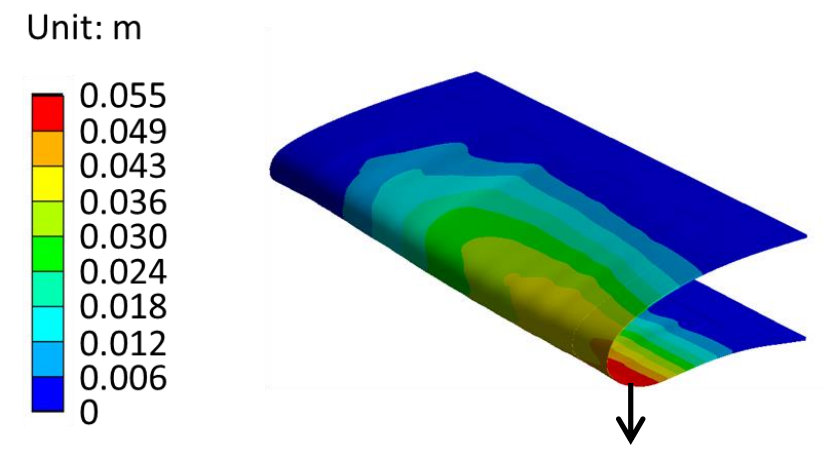

Figure 8: TOTAL DISPLACEMENT OF TRANSITION SKIN

\section{SIMULATION RESULTS FOR TRIANGLE SKIN}

The covering skin of the triangle at the aileron has other requirements, which are evaluated in the measurement. One essential requirement is the buckling of the skin during the aileron motion. Local buckling effects of the skin can occur due to the discontinuous arrangement of the fibers in the EPDM matrix. The buckling will be perpendicular to the skin surface. Figure 9 shows the deformation of the triangle and mark the displacement of the skin perpendicular to the skin surface. The results are calculated in ABAQUS as Finite Element software.

The black lines in Figure 9 represent the initial position of the triangle skin. Equal forces at two edges of the triangle are the boundary condition in the simulation model. This leads to the deformation of the triangle. The tip of the triangle does not move due to the two indicated forces. Here is a fixed bearing in the simulation.

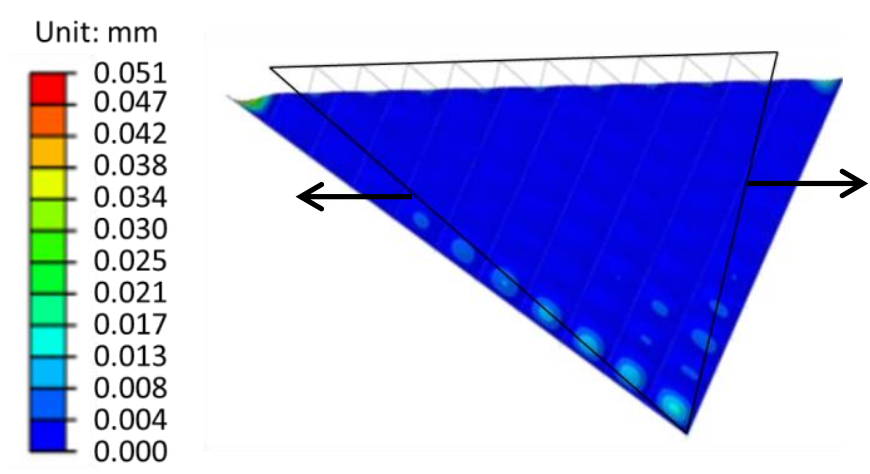

Figure 9: DEFORMATION OF SKIN PERPENDICULAR TO SURFACE

Only the skin is simulated in the ABAQUS model and the core as supporting structures is missing. However, the maximal deformation due to the tension of the triangle is only 0.05 millimeter in maximum. This is the expected maximal deformation for the experiment and occurs only in the border areas of the skin.

\section{EXPERIMENTAL INVESTIGATION}

The optical system ATOS from GOM company is used as measurement system in order to scan the demonstrator surface. The system is complete contact less and can achieve accuracies in millimeter range depending on the measurement distance. The demonstrator needs to be prepared with markers, which are glued to the surface. These markers are white in the middle and have a black ring at the outside and act as reference points for the system. Figure 10 shows the experimental setup, where the markers can be identified at the demonstrator surface. These reference points are required, because the cameras cannot capture the complete demonstrator. The measurement of the demonstrator is done in several scans in order to achieve the complete deformation. This is especially necessary for the transition skin at the leading edge, because the deformation of the skin has to be scanned from the suction and the pressure side. The software traces the reference points and combines the different scans to a complete model of the demonstrator. Another preparation step is the spraying of the demonstrator surface with removable marker paint. This is necessary on black surfaces, because the rubber parts do not reflect the light sufficient.

The ATOS system emits a precise fringe pattern to the surface. The pattern is a blue light with different black stripes in-between. A stereo camera system captures the narrow-band blue light, so that the ambient light can be filtered in the image acquisition. In the measurement only a static analyses is possible due to the projected fringe pattern. Therefore, only the initial and deflected states of the demonstrator can be captured. 


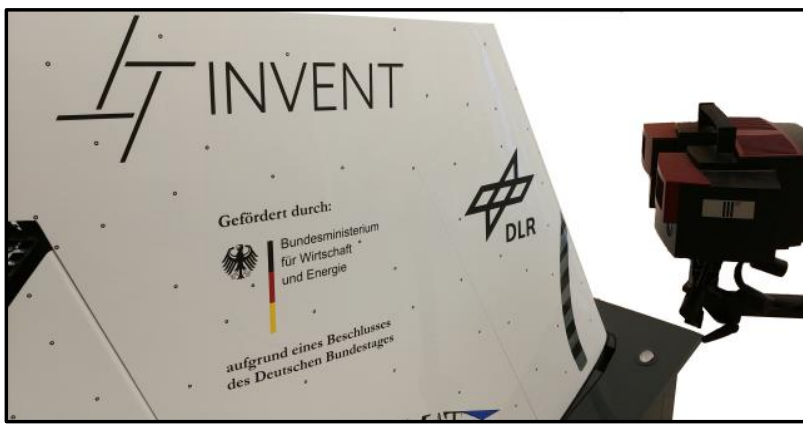

Figure 10: CLOSE-UP VIEW OF DEMONSTATOR WITH ARBITRARY DISPLACED REFERENCE DOTS AND ATOS MEASUREMENT HEAD ON THE RIGHT

Figure 11 shows the points from the ATOS measurement and the simulation of the transition skin in the initial position. A comparison approach is the calculation of the minimal distance between the points of both shapes. The simulation model consists of approximately 9000 points where the maximal distance is nine millimeter. The experimental shape consists of 1.2 million points with a distance of less than 0.01 millimeter between the points.

\section{$\times$ Simulation Experiment}

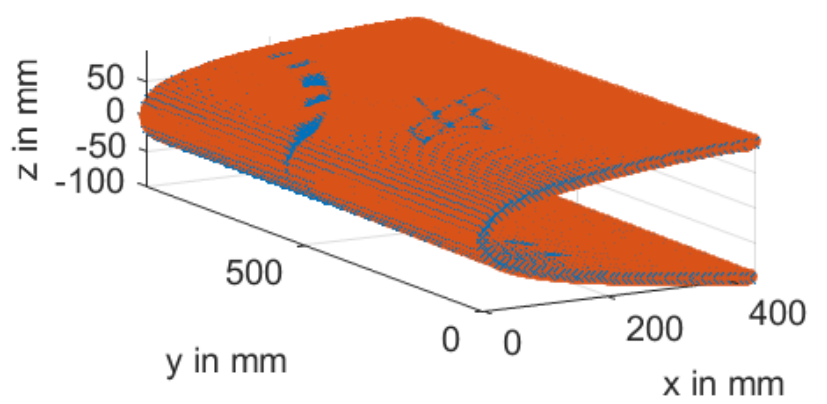

Figure 11: POINTS FROM ATOS MEASURMENT AND SIMULATION MODEL FOR INITIAL POSITION

Figure 12 shows the minimal distance between the shapes as color map on the points of the experimental shape. The distances are like a regularly pattern with a maximum distance between the simulation and experimental shapes of approximately seven millimeters. The maximum difference occurs at the span wise edges of the shapes. The pattern in Figure 12 shows an accurate manufacturing of the transition skin for the leading edge.
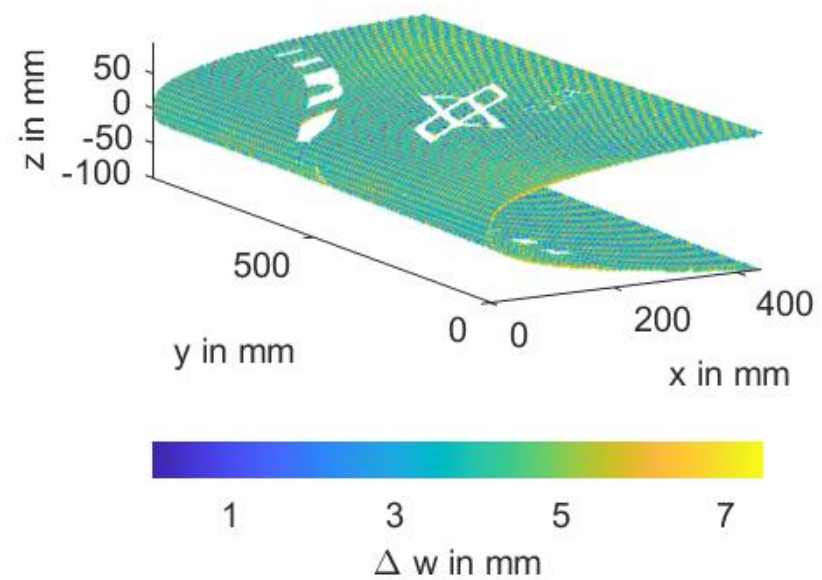

Figure 12: MINIMAL DISTANCE BETWEEN INITIAL SHAPE FROM SIMULATION AND EXPERIMENT AS COLOR MAP ON EXPERIMENTAL SHAPE

The comparison procedure between simulation and experiment is repeated with the deformed shape of the leading edge skin. The maximal deflection at the leading edge cannot be achieved like in Figure 8 due to the painting on the demonstrator. The painting does not withstand the strains. Therefore, the deformation in the simulation is reduced to a maximum of 30.5 millimeters instead of 55 millimeters. Figure 13 shows the points from the experiment and the simulation for the reduced deformation.

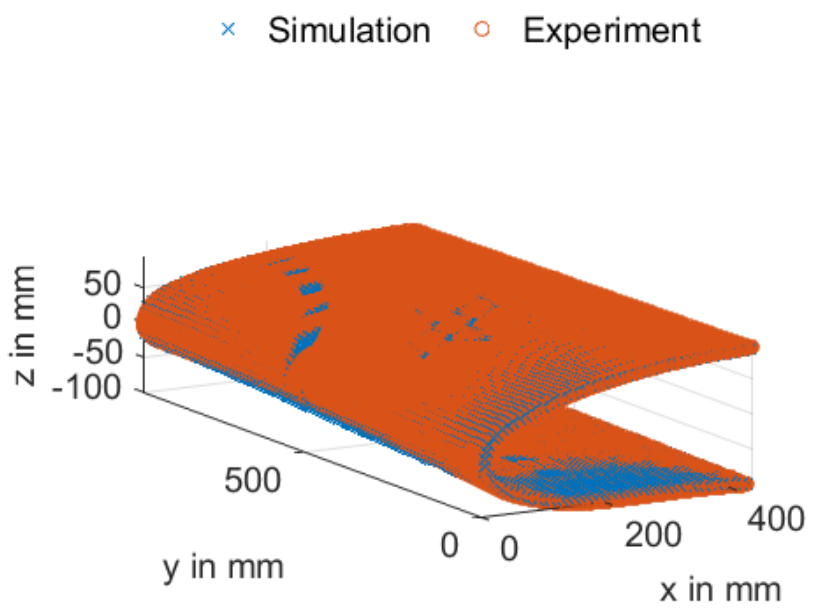

Figure 13: POINTS FROM ATOS MEASUREMENT AND SIMULATION MODEL FOR DEFORMED SHAPE

The deviation between the deformed shapes is shown in Figure 14. This figure shows the minimal distance between simulation and experiment as a color map on the points from 
the measurement. The maximal deviation is approximately 13 millimeters. This distance occurs at the root section of the profile. The contour of the demonstrator is similar to the simulation results. In order to compare the root section in more detail Figure 15 shows the 2D section plot of the simulation shape and the experimental shape.
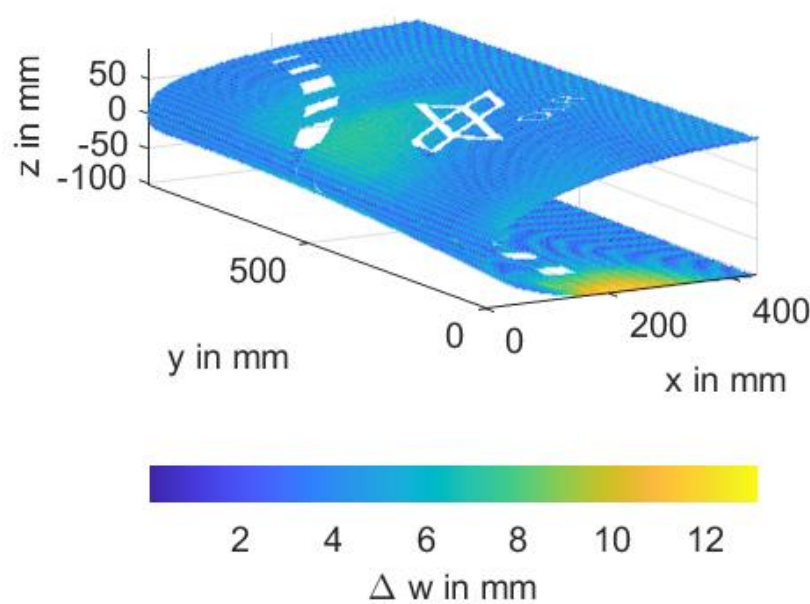

Figure 14: MINIMAL DISTANCE BETWEEN DEFORMED SHAPES FROM SIMULATION AND EXPERIMENT AS COLOR MAP ON EXPERIMENTAL SHAPE

In the experiment the shape at the pressure side is nearly flat while in the simulation a certain curvature can be observed. The result is a shape deviation of 13 millimeters. One reason could be the boundary condition at the spar. In the simulation is the fixation tighter than at the demonstrator where the skin is connected with screws to the spar.

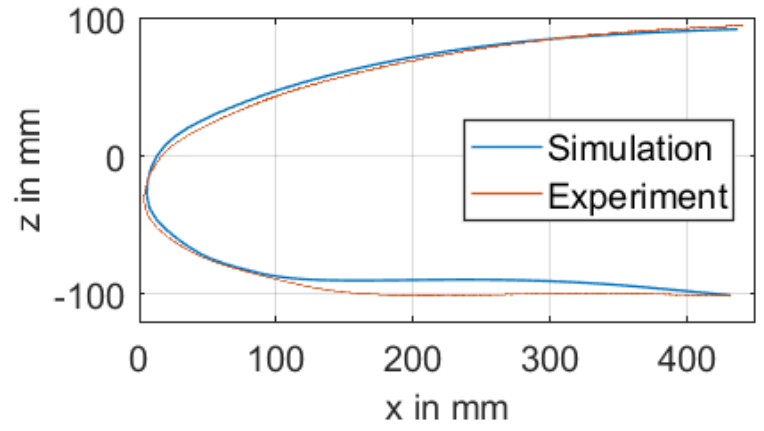

Figure 15: SIMULATION SECTION AND EXPERIMENTAL SECTION OF WING ROOT

The evaluation of the deformation for the triangle concept is much harder. Figure 16 shows the measurement points from the triangle during the aileron motion. For the measurements the demonstrator is the reference geometry, which can be positioned between the scans. The evaluation of the deformation requires a transformation of the different triangle positions in order to get out of plane deformation of the triangles. Furthermore the triangle extends during the aileron motion, which makes the orientation of the shapes more difficult, because there are no reference marks to align the shapes.

\section{$\times$ Aileron inital Aileron down * Aileron up}

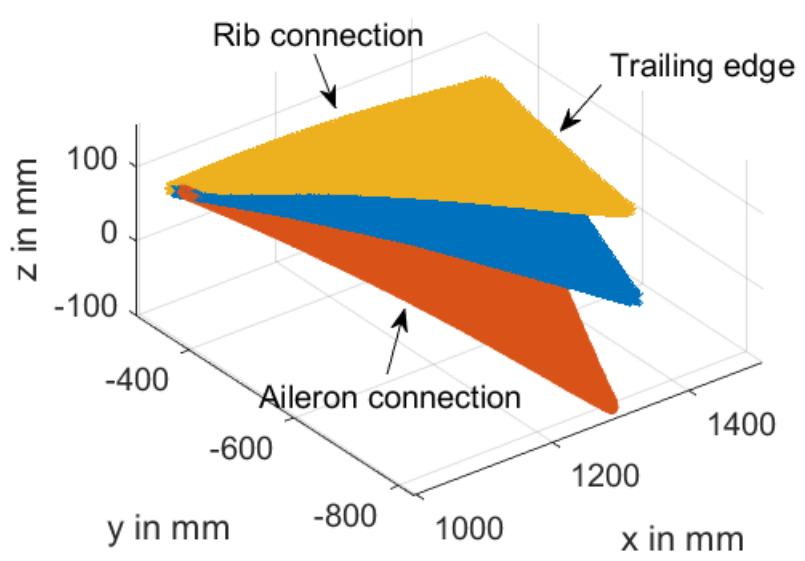

Figure 16: POINTS FROM ATOS MEASUREMENT FOR TRIANGLE IN INTIAL POSITION, AILERON DOWNWARD POSITION AND AILERON UPWARD POSITION

The approach is to select three points in the $\mathrm{x}-\mathrm{y}$-plane for each shape at the same $\mathrm{x}$ - and $\mathrm{y}$-position. These three points spans a plane for each triangle. The next step is to rotate all shapes that the normal vector of the planes is parallel to the $\mathrm{z}$ axis. Figure 17 shows the top view of the oriented triangles and the three selected points in the $x-y$-plane. The shapes are also translated that one plane point is the origin of the coordinate system.

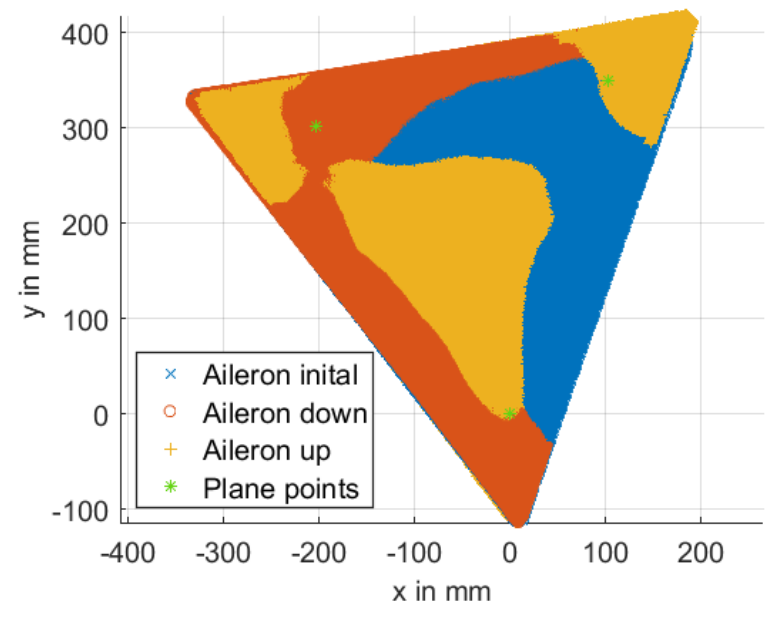

Figure 17: TRANSFORMED POINTS FROM ATOS MEASUREMENT FOR TRIANGLE AS TOP VIEW 
Now, the minimal distances are calculated between the point of the aileron up position and the initial position as well as the aileron down position and the initial position. Figure 18 shows the minimal difference between aileron down and initial position. The color bar is modified so that the yellow limit shows a deviation of 10 millimeters or more. This is necessary, because the edges of the triangle deviate most. The deviation occurs due to the selected points from the measurement. The edges of the triangle have a radius, which complicates the selection of the same points of the mesh in the measurement. Also, the triangle extends due to the aileron motion. This extension is also part of the high deviation at the triangle edges. Figure 18 shows no local buckling effects, but the results cannot be compared to the simulation in Figure 9, because the simulated out of plane deformation has a maximum of 0.05 millimeters. The experiment shows approximately six millimeter deviation between the shapes at the trailing edge. This is much higher than the simulation results and therefore the experiment cannot be compared to the experimental results at this point. A slight transformation error leads to a higher deviation in the shapes than the simulated out of plane deformation.

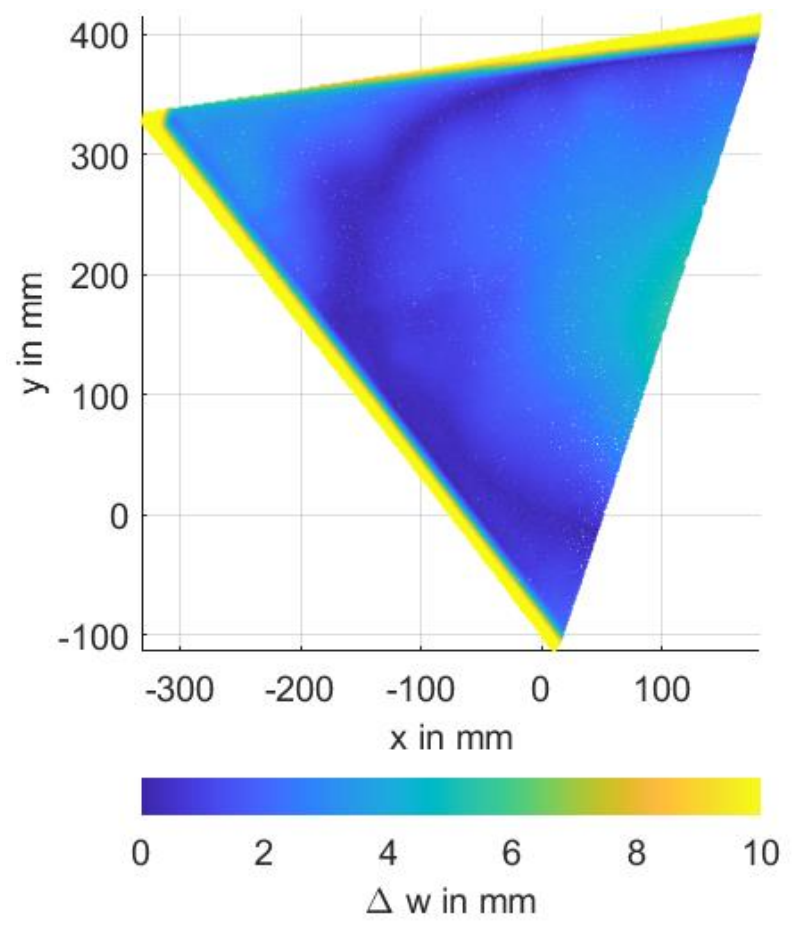

Figure 18: MINIMAL DISTANCE BETWEEN INITIAL SHAPE AND AILERON DOWNWARD MOTION

Figure 19 shows minimal difference between aileron up and initial position. Here the color bar is also in the same range with yellow equal to 10 millimeters or more. Also the edges show the highest deformation due to the different sizes of the triangle for the measurements. The maximum is by eight millimeters for this configuration. This is also much higher than the simulated out of plane deformation from Figure 9. Therefore an evaluation is not possible between simulation and experiment.

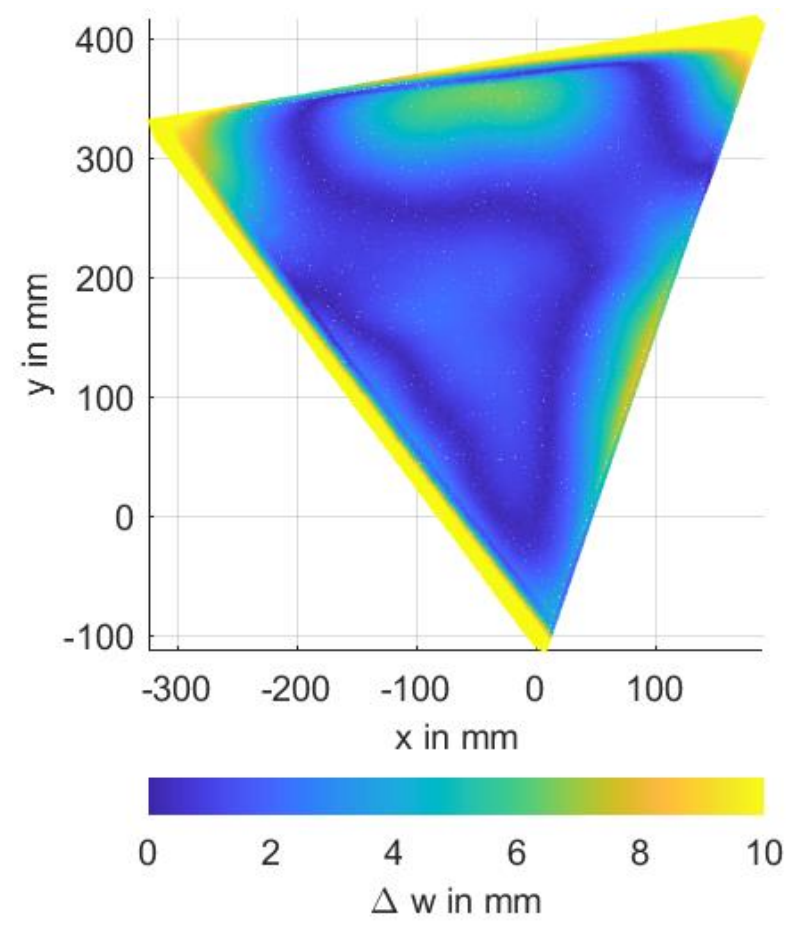

Figure 19: MINIMAL DISTANCE BETWEEN INITIAL SHAPE AND AILERON UPWARD MOTION

Even if the triangle concept cannot be compared to the simulation directly the triangle concepts seems reliable, because the experimental data did not show any local buckling effects. Therefore an adaption of the measurement procedure is required in order to observe the out of plane deformation. One possibility is the usage of reference points on the skin in order to track the motion and generate a reference plane for an improved transformation.

\section{CONCLUSION}

Two concepts for a gap cover at an aircraft wing were realized with a demonstrator. The first concept closes the gap between a droop nose and the fixed rib over a span of one meter. The second concept covers the gap between an aileron and a fixed rib in form of a triangle. The surface of the demonstrator was analyzed with an optical measurement system in the initial position and in the deflected position. The leading edge concept shows a good agreement to the simulation model and deviate in the initial position by seven millimeters and in the deflected position by 13 millimeters near the root section of the demonstrator. The comparison of the triangle between simulation and experiment reveal a challenge. The out 
of plane deflection in the simulation has a maximum of 0.05 millimeters while in the experiment the shapes deviate in the different positions up to eight millimeters. Both concepts show no excessive local deformations and they are promising for wind tunnel testing as a next step.

\section{ACKNOWLEDGEMENTS}

Thanks go to the Federal Ministry of Economic Affairs and Energy Germany for supporting the work under the project funding reference number $20 \mathrm{~W} 1507 \mathrm{C}$ and the project name FlexMat as a part of its aviation research program under contract LUFOV2-790-305.

\section{REFERENCES}

[1] Brooks, Thomas F. and Humphreys, William M. "Flapedge aeroacoustic measurements and predictions." Journal of Sound and Vibration Vol. 261 No. 1 (2003): pp. 31-74. DOI 10.1016/S0022-460X(02)00939-2.

[2] Kota, Sridhar, Osborn, Russell, Ervin, Gregory, Maric, Dragan, Flick, Peter, and Paul, Donald. "Mission adaptive compliant wing--design, fabrication and flight test.". RTO Applied Vehicle Technology Panel (AVT) Symposium 2009. Evora, Portugal.

[3] Hutcheson, Florence V., Brooks, Thomas F., and Humphreys, William M. "Noise Radiation from a Continuous Mold-Line Link Flap Configuration." International Journal of Aeroacoustics Vol. 10 5-6 (2011): pp. 565-588. DOI 10.1260/1475-472X.10.5-6.565.

[4] Woods, Benjamin K.S., Parsons, Laura, Coles, Alexander B., Fincham, James H.S., and Friswell, Michael I. "Morphing elastically lofted transition for active camber control surfaces." Aerospace Science and Technology Vol. 55 (2016): pp. 439-448. DOI 10.1016/j.ast.2016.06.017.

[5] Barbarino, Silvestro, Bilgen, Onur, Ajaj, Rafic M., Friswell, Michael I., and Inman, Daniel J. "A Review of Morphing Aircraft." Journal of Intelligent Material Systems and Structures Vol. 22 No. 9 (2011): pp. 823877. DOI 10.1177/1045389X11414084.

[6] Vasista, Srinivas, Tong, Liyong, and Wong, K. C. "Realization of Morphing Wings: A Multidisciplinary Challenge." Journal of Aircraft Vol. 49 No. 1 (2012): pp. 11-28. DOI 10.2514/1.C031060.

[7] Kintscher, Markus, Wiedemann, Martin, Monner, Hans Peter, Heintze, Olaf, and Kühn, Timo. "Design of a smart leading edge device for low speed wind tunnel tests in the European project SADE." International Journal of Structural Integrity Vol. 2 No. 4 (2011): pp. 383-405. DOI 10.1108/17579861111183911.

[8] Caton, John H., Hobey, Michael J., Groeneveld, John D., Jacobs Jack H., Wille, Robert H., and Brase, Lawrence O. JR. “CONTROL SURFACE FOR AN AIRCRAFT: United States Patent Application Publication.” US Patent 2001/0006207 A1 (2001).

[9] Diller, Joseph B. and Miller, Nicholas F. JR. "ELASTOMERIC TRANSTION FOR AIRCRAFT
CONTROL SURFACE: United States Patent." US Patent 6,145,791 (1998).

[10] Etling, Keith A. "MORPHING CONTROL SURFACE TRANSTION." US Patent 8,342.447 B2 (2010).

[11] Khorrami, Mehdi R., Lockard, David P., Moore, James B., Su, Ji, Turner, Travis L., Lin, John C., Taminger, Karen M., Kahng, Seun K., and Verden Scott A. "ELASTICALLY DEFORMABLE SIDE-EDGE LINK FORTRALING-EDGE FLAP AEROACOUSTIC NOISE REDUCTION: United States Patent." US Patent 8,695,925 B2 (2011).

[12] Kunz, Rüdiger. “APPARATUS FOR CLOSING AN AIR GAP BETWEENA FLAP AND AN AIRCRAFT: United States Patent." US Patent 4,471,925 (1982).

[13] Khorrami, Mehdi R., Humphreys, William M., Lockard, David P., and Ravetta, Patricio A. "Aeroacoustic Evaluation of Flap and Landing Gear Noise Reduction Concepts.". AIAA Aviation: 20th AIAA/CEAS Aeroacoustics Conference. Atlanta, GA. DOI 10.2514/6.2014-2478.

[14] Sreekantamurthy, Tham, Turner, Travis L., Moore, James B., and Su, Ji. "Elastomeric Structural Attachment Concepts for Aircraft Flap Noise Reduction - Challenges and Approaches to Hyperelastic Structural Modeling and Analysis.". 55th AIAA/ASME/ASCE/AHS/ASC Structures, Structural Dynamics, and Materials Conference. National Harbor, Maryland. DOI 10.2514/6.2014-0509.

[15] Streett, Craig, Casper, Jay, Lockard, David, Khorrami, Mehdi, Stoker, Robert, Elkoby, Ronen, Wenneman, Wayne, and Underbrink, James. "Aerodynamic Noise Reduction for High-Lift Devices on a Swept Wing Model.". 44th AIAA Aerospace Sciences Meeting and Exhibit. Reno, Nevada, 09 January 2006 - 12 January 2006. DOI 10.2514/6.2006-212.

[16] Storms, Bruce, Jaeger, Stephen, Hayes, Julie, and Soderman, Paul. "Aeroacoustic study of flap-tip noise reduction using continuous moldline technology.". 6th Aeroacoustics Conference and Exhibit. Lahaina,HI,U.S.A, 12 June 2000 - 14 June 2000. DOI 10.2514/6.2000-1976.

[17] Dargel, G., Hansen, H., Wild, J., Streit, T., Rosemann, H., and Richter, K. "Aerodynamische Flügelauslegung mit Multifunktionalen Steuerflächen.”. Deutscher Luft- und Raumfahrtkongress 2002. Stuttgart, 23.9.-26.9.

[18] da Rocha-Schmidt, L. and Baier, H. "Morphing Skins and Gap Covers for Aerodynamic Control Surfaces.". 62. Deutscher Luft- und Raumfahrtkongress 2013. Stuttgart, Germany, 10.-12.September.

[19] da Rocha-Schmidt, L. and Baier, H. "A Shape Variable Gap Cover Concept for Aerodynamic Control Surfaces Based on Shear Deformation.". 63. Deutscher Luft- und Raumfahrtkongress 2014. Augsburg, Germany, 16.-18. September.

[20] Krollmann, J., Neuchl, S., Schaefer, P.M., Achleitner, J., and Drechsler, K. "Hybrid-matrix composites: materials and manufacturing.". SAMPE 2017 - Society of the 
Advancement Material and Process Engineering. Seattle, 22-25 May.

[21] Krollmann, Jan Wolfgang. A contribution to integral fiber reinforced hinges based on carbon fiber reinforcd

elastomers and the hybrid-matrix approach. Verlag Dr.

Hut, München (2018).

[22] Radestock, Martin, Falken, Alexander, Riemenschneider, Johannes, and Kintscher, Markus. "Hybrid Skin Design of the Transition Region Between Morphing Wing and Fixed Wing.". ASME 2018 Conference on Smart Materials, Adaptive Structures and Intelligent Systems: Volume 1: Development and Characterization of Multifunctional Materials; Modeling, Simulation, and Control of Ada. San Antonio, Texas, USA, 9/10/2018 - 9/12/2018. DOI 10.1115/SMASIS2018-7976. 